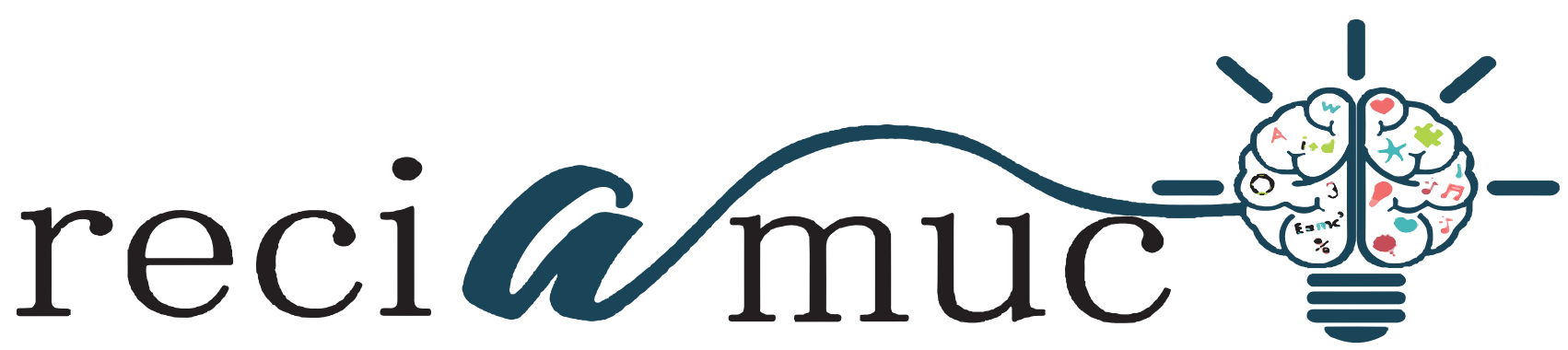

DOI: 10.26820/reciamuc/4.(3).julio.2020.352-359

URL: https://reciamuc.com/index.php/RECIAMUC/article/view/533

EDITORIAL: Saberes del Conocimiento

REVISTA: RECIAMUC

ISSN: 2588-0748

TIPO DE INVESTIGACIÓN: Articulo de Investigación

Código UNESCO: 32 Ciencias Médicas; 3201 Ciencias Clínicas

PAGINAS: 352-359

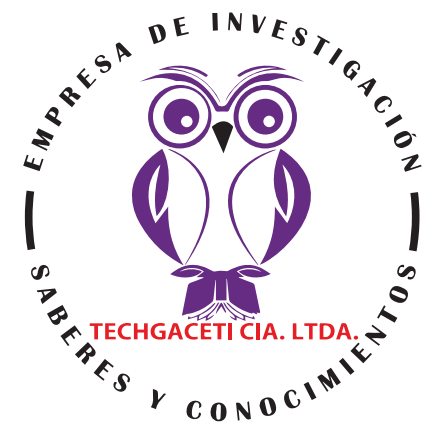

\title{
Síndrome de la vena cava superior
}

Superior vena cava syndrome

Síndrome da veia cava superior

\section{Liliana Elizabeth Flores Rodríguez'; Danny Fernando Tobar Durán2; Bryan Rolando Tutin Miniguano3; Adriana Malena Cueva Loaiza ${ }^{4}$}

RECIBIDO: 20/05/2020 ACEPTADO: 19/07/2020 PUBLICADO: 30/07/2020

1. Médico General; Hospital Carlos Andrade Marín; Quito, Ecuador; dr.liliana.flores@gmail.com; (DD https://orcid.org/00000002-8303-4411

2. Médico; Hospital Carlos Andrade Marín; Quito, Ecuador; dannytobar14@gmail.com; (iD https://orcid.org/0000-0001-78777467

3. Médico; Hospital Carlos Andrade Marín; Quito, Ecuador; bryan_rtm@hotmail.com; (iD https://orcid.org/0000-0001-52214615

4. Médico; Hospital Pediátrico Vaca Ortiz; Quito, Ecuador; cuevaadriana94@gmail.com; (D) https://orcid.org/0000-0002-73736225

CORRESPONDENCIA

Liliana Elizabeth Flores Rodríguez

dr.liliana.flores@gmail.com

Quito, Ecuador 


\section{RESUMEN}

EI SVCS se genera por la obstrucción parcial o completa del flujo sanguíneo, la evolución de los estudios en los últimos 50 años ha sido importante en cuanto a su etiología, ya que en los primeros años de su descubrimiento, esta se presentaba por infecciones, dando paso en la actualidad a procesos tumorales. La metodología de la investigación es una revisión bibliográfica, apoyada en recursos electrónicos para su desarrollo. Las principales conclusiones son que las mayores causas que producen este síndrome es el cáncer de pulmón; los estudios imagenologicas son fundamentales para su detección, entre los tratamientos más recomendados para el abordaje de esta patología es la prótesis autoexpandible intravascular y entre las principales causas que la originan, pueden ser malignas y benignas.

Palabras clave: SVCS, Pulmón, Cáncer, Radioterapia, Imagenologica.

\section{ABSTRACT}

SVCS is generated by partial or complete obstruction of blood flow, the evolution of studies in the last 50 years has been important in terms of its etiology, since in the first years of its discovery, it was presented by infections, giving I currently go to tumor processes. The research methodology is a bibliographic review, supported by electronic resources for its development. The main conclusions are that the main causes that produce this syndrome is lung cancer; Imaging studies are essential for its detection, among the most recommended treatments for the approach of this pathology is the intravascular self-expanding prosthesis and among the main causes that originate it, they can be malignant and benign.

Keywords: SVCS, Lung, Cancer, Radiotherapy, Imaging.

\section{RESUMO}

A SVCS é gerada por obstrução parcial ou total do fluxo sanguíneo, a evolução dos estudos nos últimos 50 anos tem sido importante em termos de sua etiologia, já que nos primeiros anos de sua descoberta era apresentada por infecções, dando atualmente a processos tumorais. A metodologia da pesquisa é uma revisão bibliográfica, apoiada em recursos eletrônicos para o seu desenvolvimento. As principais conclusões são que as principais causas que produzem essa síndrome são o câncer de pulmão; Os estudos de imagem são essenciais para a sua detecção, entre os tratamentos mais recomendados para a abordagem desta patologia está a prótese autoexpansível intravascular e entre as principais causas que a originam podem ser malignas e benignas.

Palavras-chave: SVCS, Lung, Cancer, Radiotherapy, Imaging. 


\section{Introducción}

En 1749 William Hunter describió este síndrome en un paciente con un aneurisma de aorta sifilítico; durante muchos años las infecciones fueron la principal causa del SVCS. Posteriormente, la etiología infecciosa ha sido desplazada por los procesos tumorales, siendo el más frecuente el cáncer de pulmón. En los últimos años ha surgido como una nueva causa la obstrucción de la VCS secundaria al uso de catéteres endovenosos (para quimioterapia, hemodiálisis, marcapasos o desfibriladores) (Muñiz, Arregui, Hodge, Vallenilla, \& Merino, 2015).

El síndrome de vena cava superior (SVCS), es el conjunto de síntomas y signos derivados de la obstrucción parcial o completa del flujo sanguíneo a través de la vena cava superior hacia la aurícula derecha. Puede ser debido a trombosis intrínseca (idiopática o primaria) o a la compresión extrínseca (asociada o no a trombosis secundaria). Constituye una de las escasas situaciones de urgencia médica en Oncología, por lo que su diagnóstico precoz es clave para un eficaz tratamiento y un mejor pronóstico a medio plazo (GARCÍA, y otros, 2001).

El manejo de estos enfermos presenta siempre grandes dificultades en ocasiones como grave urgencias clínicas. La evolución etiológica en estos últimos 50 años ha presentado grandes variaciones. La cavografía superior se ha considerado durante muchos años como un dato fundamental para el diagnóstico y la pauta terapéutica sin embargo, últimamente este criterio se ha sometido a grandes polémicas. El tratamiento utilizado, las respuestas obtenidas y la distinción entre el pronóstico del SVCS y de la enfermedad causal son actualmente motivo de discusión (Pila Pérez, Pila Peláez, del Sol Sosa, Paulino Basulto, \& Arteaga Ocaña, 2002).

La VCS es el principal conducto para el drenaje venoso de la cabeza, cuello, las extremidades superiores y el tórax superior, se trata de un vaso de pared fina, blando, loca- lizado en el mediastino medio y rodeado de estructuras más rígidas, como los ganglios mediastínicos y paratraqueales, la tráquea y el bronquio principal derecho, la arteria pulmonar y la aorta. Se extiende desde la unión de las venas innominadas derecha e izquierda a la aurícula derecha, por una distancia de 6 a $8 \mathrm{~cm}$, los $2 \mathrm{~cm}$ distales de la VCS se encuentran dentro del saco pericárdico y en la reflexión de este se produce cierta fijación de la VCS, la anchura de la VCS es de 1.5 a 2 cm (Arce \& Cortés, 2015).

Tabla 1. Signos y síntomas asociados con SVCS

\begin{tabular}{|lcc|} 
Signo o Síntoma & $\begin{array}{c}\text { Porcentaje } \\
\text { de } \\
\text { todos los } \\
\text { pacientes }\end{array}$ & $\begin{array}{c}\text { Rango } \\
(\%)\end{array}$ \\
\hline Hemodinámicas & 82 & $60-100$ \\
\hline Edema facial & 46 & $14-75$ \\
\hline Edema en brazos & 63 & $27-86$ \\
\hline $\begin{array}{l}\text { Distención de venas } \\
\text { del cuello }\end{array}$ & 53 & $38-67$ \\
\hline $\begin{array}{l}\text { Distención de venas } \\
\text { del pecho }\end{array}$ & 20 & $13-23$ \\
\hline Plétora Facial & 2 & - \\
\hline Síntomas visuales & & \\
\hline Respiratorios & 54 & $23-74$ \\
\hline Disnea & 54 & $38-70$ \\
\hline Tos & 17 & - \\
\hline Voz ronca & 4 & - \\
\hline Estridor & & \\
\hline Neurológicos & 10 & $8-13$ \\
\hline Síncope & 9 & $6-11$ \\
\hline Cefalea & 6 & $2-10$ \\
\hline Mareo & 4 & - \\
\hline Confusión & 2 & - \\
\hline $\begin{array}{l}\text { Obnubilación/evento } \\
\text { cerebro vascular }\end{array}$ & & \\
\hline & & \\
\hline
\end{tabular}

Fuente: (Alvarado Arce \& Odio Cortés, 2015). 


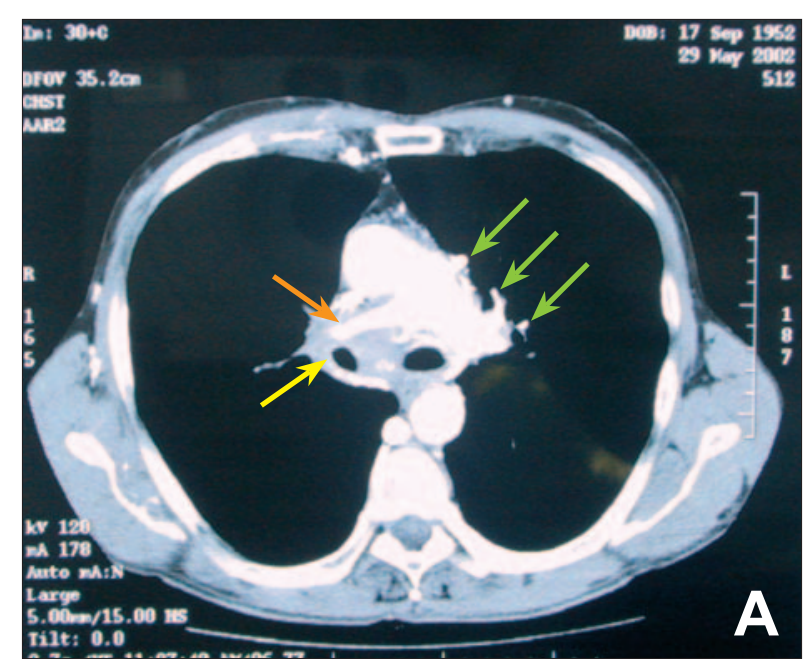

Figura 1. Disminución de calibre de la VCS (flecha amarilla) y arteria pulmonar (flecha naranja). Presencia de circulación colateral (flechas verdes)

Fuente: (Fernández Jorge, y otros, 2004).

El diagnóstico de sospecha es clínico y se confirma con pruebas de imagen (radiografía de tórax, TC con contraste, RNM). Las manifestaciones cutáneas pueden ser la forma de presentación de este síndrome y deben inducirnos a buscar una neoplasia oculta. Exponemos dos casos de SVCS diagnosticados a partir de dichas manifestaciones cutáneas (Fernández Jorge, y otros, 2004).

\section{Metodología}

La metodología de la investigación, es una revisión bibliográfica, que ha sido apoyada mediante recursos electrónicos, en donde se han encontrado muchos trabajos que hacen referencia acerca del síndrome de la vena cava superior, lo que se busca con la presente investigación, es hacer referencia al diagnóstico, causas y tratamiento, de esta patología.

\section{Resultados}

La altura de la obstrucción determina en gran parte el cuadro clínico y su velocidad de instauración:

- Si afecta a la vena cava proximal (craneal a la entrada de la ácigos), el síndrome es menos pronunciado, ya que el sistema ácigos se distiende rápida y fácilmente, reduciendo la presión venosa en los territorios superiores del cuerpo. La circulación colateral a través de las venas mamarias internas y costoaxilares no es apreciable en la pared torácica, pero da lugar a distensión visible de la vena yugular derecha.

- Si se localiza en la vena cava distal (caudal a la entrada de la ácigos), el cuadro clínico es más manifiesto; el retorno venoso a través de las venas abdominales superiores y la vena cava inferior produce circulación colateral toraco-abdominal visible y, en casos más graves, edema de miembros inferiores e incluso ascitis (Muñiz, Arregui, Hodge, ValleniIla, \& Merino, 2015).

Lo habitual es que la instauración del cuadro clínico sea progresiva a lo largo de varias semanas (alrededor de 2 semanas en un tercio de los pacientes y durante periodos más largos en el resto). Normalmente, el síntoma más frecuente y precoz es la disnea, seguido de la tríada clásica: edema en esclavina, cianosis facial y circulación colateral (Muñiz, Arregui, Hodge, Vallenilla, \& Merino, 2015).

Es típico que los síntomas empeoren al in- 
clinarse hacia delante y al tumbarse, por lo que los signos clínicos suelen ser más evidentes por la mañana. El aumento de la cianosis, el edema facial y la congestión cefálica al elevar los brazos por encima de la cabeza, se conoce como signo de Botermann (Muñiz, Arregui, Hodge, Vallenilla, \& Merino, 2015).

Tabla 2. Principales causas malignas del síndrome de la vena cava superior

\begin{tabular}{|l|c|}
\hline Tipo de tumor & $\begin{array}{c}\text { Proporción, } \\
\% \text { (intervalo) }\end{array}$ \\
\hline Cáncer pulmonar de células no pequeñas & $50(43-59)$ \\
\hline Cáncer pulmonar de células pequeñas & $22(7-39)$ \\
\hline Linfoma & $12(1-25)$ \\
\hline Cáncer metastásico & $9(1-15)$ \\
\hline Cáncer de células germinales & $3(0-6)$ \\
\hline Timoma & $2(0-4)$ \\
\hline Mesotelioma & $1(0-1)$ \\
\hline Otros tipos de cáncer & $1(0-2)$ \\
\hline
\end{tabular}

Fuente: (Pech-Alonso, Arredondo-Ruiz, González-Galván, \& Fermín-Hernández, 2018).

Tabla 3. Propuesta de clasificación del SVCS según signos y síntomas

\begin{tabular}{|c|c|c|c|}
\hline Grado & Categoría & $\begin{array}{l}\text { Incidencia } \\
\text { estimada } \\
(\%)\end{array}$ & Definición* \\
\hline $\mathbf{0}$ & Asintomático & 10 & Obstrucción radiográfica de la VCS en ausencia de síntomas. \\
\hline 1 & Leve & 25 & Edema en cabezo o cuello (distención vascular), cianosis, plétora. \\
\hline 2 & Moderado & 50 & $\begin{array}{l}\text { Edema en cabeza o cuello con discapacidad funcional (disfagia leve, } \\
\text { tos, leve o moderada limitación al movimiento de la cabeza, mandíbula } \\
\text { o movimientos oculares, molestias visuales debido al edema ocular). }\end{array}$ \\
\hline 3 & Severo & 10 & $\begin{array}{l}\text { Leve o moderado edema cerebral (cefalea, mareos) o leve/moderado } \\
\text { edema laríngeo o disminución de la reserva cardiaca (sincope). }\end{array}$ \\
\hline 4 & $\begin{array}{l}\text { Amenaza la } \\
\text { vida }\end{array}$ & 5 & $\begin{array}{l}\text { Edema cerebral significativo (confusión, obnubilación) o edema larín- } \\
\text { geo significativo (estridor) o compromiso hemodinámico significativo } \\
\text { (síncope sin factores precipitantes, hipotensión, insuficiencia renal). }\end{array}$ \\
\hline 5 & Fatal & $<1$ & Muerte. \\
\hline \multicolumn{4}{|c|}{$\begin{array}{l}\text { "Cada signo o síntoma debe deberse a una obstrucción de la VCS y los efectos del edema cerebral o laríngeo o los efectos en } \\
\text { la función cardiaca. Los síntomas causados por otros factores (parálisis de cuerdas vocales, compromiso del árbol traqueo } \\
\text { bronquial o el corazón por efecto de masa) no deben considerarse ya que se deben a un efecto de masa en otros órganos y no a } \\
\text { una obstrucción de la VCS. }\end{array}$} \\
\hline
\end{tabular}

Fuente: (Alvarado Arce \& Odio Cortés, 2015)

\section{Causas}

Maligna: la más frecuente, pudiendo ser neoplasias o metástasis con afectación mediastínica.

El cáncer es la causa más frecuente de SVCS. El cáncer de pulmón, de célula pequeña o grande, puede invadir directamente la VCS. El linfoma, Hodgkin o no Hodgkin, tiende a comprimir el vaso. Otros tumores mediastínicos, incluyendo el timoma maligno, el tumor germinal y las metástasis o tumores pleurales, como el mesotelioma, también pueden causar SVCS.

La mayoría de tumores malignos de la vena cava, como el leiomiosarcoma o angiosarcoma, afectan a la VCl. La afectación de la VCS es muy rara. Si existe realce arterial del trombo es muy sugestivo de trombo tumo- 
ral (Cerdan, Vara, Auñón, Weitz, \& Lozano, 2012).

Benigna: incluye infecciones, mediastinitis fibrosante, bocio tiroideo endotorácico, aneurisma aórtico, tumores benignos, hematoma mediastínico, sarcoidosis o fibrosis inducida por radiación.

La mediastinitis fibrosante es una enfermedad benigna rara debida a la proliferación de tejido colágeno y a la fibrosis del mediastino. Puede ser idiopática, causada por una respuesta inmunológica anómala a una infección por Histoplasma capsulatum o por tuberculosis, o puede asociarse a una fibrosis retroperitoneal, especialmente en su forma difusa.

En el TCMD la mediastinitis fibrosante aparece como una masa de partes blandas mal definida obliterando la grasa normal. El que se afecta con más frecuencia es el mediastino medio. Existe otra forma más focal con calcificaciones.

La enfermedad de Behçet es una enfermedad sistémica rara que afecta más frecuentemente a la población del Mediterráneo, oriente medio y Asia. Causa úlceras genitales y orales recurrentes y uveítis. Es una enfermedad inflamatoria asociada a vasculitis leucocitoclástica que afecta a arterias y venas sistémicas y pulmonares. La estenosis u oclusión de la VCS en el Behçet puede ser causada por tromboflebitis o por una mediastinitis fibrosante, aunque la afectación de grandes venas no es frecuente (Cerdan, Vara, Auñón, Weitz, \& Lozano, 2012).

latrogénica: como una cateterización venosa. Esta causa ha aumentado debido al incremento del uso de catéteres intravasculares, que producen una reacción de la pared venosa como respuesta a un trauma repetido. Los catéteres venosos centrales grandes, como los catéteres de diálisis, los catéteres Hickman, los de nutrición parenteral y los cables de marcapasos cardiaco permanente transvenoso, se han visto relacionados con el SVCS (Cerdan, Vara, Auñón, Weitz, \& Lozano, 2012).

\section{Tratamiento}

El manejo del SVCS debido a una malignidad depende de la etiología del cáncer, la extensión de la obstrucción, la severidad de los síntomas y el pronóstico del paciente. La expectativa de vida de un paciente con SVCS por causa maligna tiene una media de aproximadamente 6 meses, con un rango de 1.5 a 9.5 meses, pero esto varía dependiendo del tipo de cáncer. El manejo debe ir orientado a descomprimir la VCS y al tratamiento de la malignidad (Alvarado Arce \& Odio Cortés, 2015).

El tratamiento médico de soporte se basa en tomar las siguientes medidas: elevación de la cabecera de la cama, administración de oxígeno, corticosteroides y diuréticos (Alvarado Arce \& Odio Cortés, 2015).

Los pacientes que desarrollan SVCS asociado a catéter son candidatos para terapia anticoagulante o remoción del catéter. Con la radioterapia, es probable que un $10 \%$ de los pacientes con SVCS maligno no respondan durante la primera semana, en estos casos es probable que la causa de la falta de respuesta sea un coágulo, por lo que la trombolisis se debe considerar (Alvarado Arce \& Odio Cortés, 2015).

La RT (radioterapia) es de elección en el SVCS producido por tumores no sensibles a la quimioterapia. Se recomienda iniciar con fracciones grandes, con la idea de conseguir una respuesta más rápida, aunque hay pocas pruebas en la literatura que lo justifiquen, recientemente se ha utilizado con éxito la RT estereotáctica con fracciones altas para el tratamiento del SVCS producido por carcinoma de pulmón de células no pequeñas (Alvarado Arce \& Odio Cortés, 2015).

Las prótesis autoexpandibles intravasculares son estructuras metálicas que se colocan en la luz de la VCS, dilatándola y restableciendo la permeabilidad vascular. Esta técnica se ha convertido en el tratamiento de primera línea en muchos casos: 
- Es el procedimiento de elección en los pacientes con síntomas graves, que requieren una actuación terapéutica rápida. Su correcta colocación permite una rápida restauración del flujo normal en la mayoría de los casos, con resolución inmediata de los síntomas. Permite realizar posteriormente el diagnóstico histológico y el tratamiento con quimioterapia y/o radioterapia

- También son muy útiles en el SVCS secundario a catéteres centrales que trombosan la cava. En algunos casos la trombosis se resuelve al retirar el catéter (Muñiz, Arregui, Hodge, Vallenilla, \& Merino, 2015).

El tratamiento del SVCS se basa en dos aspectos fundamentales: alivio de los síntomas y tratamiento específico de la causa. El primer punto es la base de actuación del médico en la urgencia hospitalaria. Se suele recomendar reposo en cama, oxigenoterapia para aliviar la disnea, dieta pobre en sal, diuréticos tipo furosemida, (aunque estos están cada vez más discutidos), para reducir el edema y corticoides a dosis altas para reducir la inflamación y aliviar la congestión y el edema. En los casos de SVCS secundario a trombosis por catéteres se pueden emplear trombolíticos tipo uroquinasa o estreptoquinasa (GARCÍA, y otros, 2001).

Dentro de las medidas paliativas de reciente introducción en el tratamiento del SVCS está el empleo de Stents, prótesis de metal autoexpandibles. Se colocan con anestesia local y bajo control radiológico, y han permitido el seguimiento de algunos pacientes entre 2-17 meses, con una media de 6 meses (GARCÍA, y otros, 2001).

\section{Conclusiones}

El síndrome de la vena cava superior, es un padecimiento que no es muy común, las principales causas pueden ser malignas o benignas, sin embargo un diagnóstico temprano es importante para establecer el mejor tratamiento.
Este síndrome tiene hasta 6 categorías, siendo la ultima la más grave, que puede implicar la muerte. El cáncer de pulmón, es el que más produce SVCS, entre un 75$80 \%$, en segundo lugar los linfomas no Hodking entre un $2-4 \%$.

Los estudios imagenologicas son fundamentales para el diagnóstico, entre ellos, la tomografía computarizada multidetector (TCMD), ya que indica el lugar de la obstrucción, su extensión y su causa. Hay diferentes tratamientos para el abordaje de esta patología y el más recomendado es la prótesis autoexpandible intravascular.

Su manifestación clínica más frecuente, es el edema facial, seguido del edema de miembros superiores, sin embargo se pueden dar otras sintomatologías como síndromes respiratorios y alteraciones neurológicas.

\section{Bibliografía}

Alvarado Arce, E., \& Odio Cortés, D. (2015). Síndrome de vena cava superior: una emergencia médico quirúrgica. Revista Clínica Escuela de Medicina UCR-HSJD, 5(1), 11-21. Obtenido de https://revistas.ucr.ac.cr/index.php/clinica/article/ view/18338/18526

Cerdan, S. A., Vara, R., Auñón, J., Weitz, A., \& Lozano, A. (2012). Síndrome de la vena cava superior: causas, fisiopatología y hallazgos radiológicos.

Fernández Jorge, B., Peña Penabad, C., Almagro, M., Rodríguez Lozano, J., Fonseca, E., \& Cageao, C. (2004). Síndrome de la vena cava superior. Medicina Cutánea Ibero-Latino-Americana, 32(4), 163-166. Obtenido de https://www.medigraphic. com/pdfs/cutanea/mc-2004/mc044e.pdf

GARCíA, B. D., Gordo, A., Saenz, E., Auñón, P., Moreira, M., \& Barón, M. (2001). Síndrome de vena cava superior: presentación de seis casos. In Anales de medicina interna, 18(7), 369-372.

Muñiz, S. H., Arregui, P., Hodge, S., Vallenilla, A., \& Merino, J. (2015). SINDROME DE LA VENA CAVA SUPERIOR: APORTACION DE LOS METODOS DE IMAGEN.

Pech-Alonso, B., Arredondo-Ruiz, P., González-Galván, L., \& Fermín-Hernández, C. (2018). Síndrome de la vena cava superior: diagnóstico y tratamiento. Medicina interna de México, 34(3), 403-411. 
Obtenido de https://www.medigraphic.com/pdfs/ medintmex/mim-2018/mim183h.pdf

Pila Pérez, R., Pila Peláez, R., del Sol Sosa, J., Paulino Basulto, M., \& Arteaga Ocaña, M. (2002). Síndrome de la vena cava superior: Comunicación de un caso y algunas consideraciones. Revista Archivo Médico de Camagüey, 6(2), 203-209.

\section{(c) (1) $\circledast(0)$ BY NC SA}

RECONOCIMIENTO-NOCOMERCIAL-COMPARTIRIGUAL CC BY-NC-SA

ESTA LICENCIA PERMITE A OTROS ENTREMEZCLAR, AJUSTAR Y CONSTRUIR A PARTIR DE SU OBRA CON FINES NO COMERCIALES, SIEMPRE $Y$ Y CUANDO LE RECONOZCAN LA AUTORÍA Y SUS NUEVAS CREACIONES ESTÉN BAJO UNA LICENCIA CON LOS MISMOS TÉRMINOS.

\section{CITAR ESTE ARTICULO:}

Flores Rodríguez, L., Tobar Durán, D., Tutin Miniguano, B., \& Cueva Loaiza, A. (2020). Síndrome de la vena cava superior. RECIAMUC, 4(3), 352-359. doi:10.26820/reciamuc/4.(3).julio.2020.352-359 